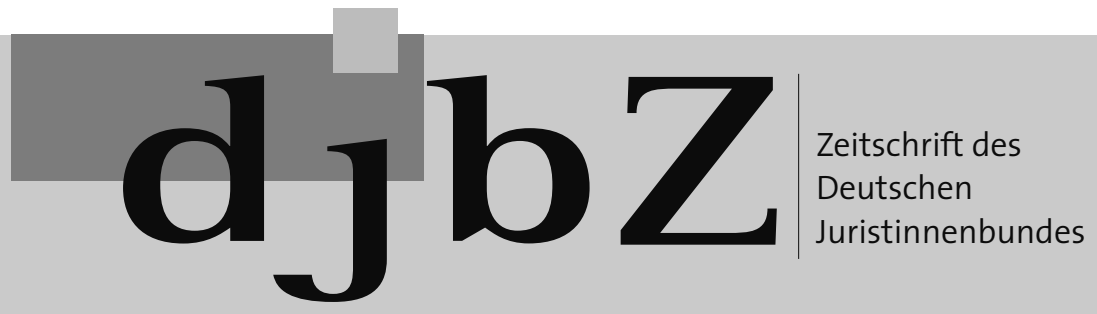

$3 / 2008$

11. Jahrgang, Seite $113-170$

Herausgeber: Deutscher Juristinnenbund e. V.

Präsidium: Jutta Wagner, Rechtsanwältin und Notarin, Fachanwältin für Familienrecht, Berlin (Präsidentin); Renate Maltry, Rechtsanwältin, Fachanwältin für Familienrecht und Erbrecht, München; Ramona Pisal, Vorsitzende Richterin am Oberlandesgericht, Brandenburg a. d. H. (Vizepräsidentinnen); Dagmar Brinkmann, Regierungsdirektorin, Frankfurt a.M. (Schatzmeisterin); Schriftleitung: Anke Gimbal, Rechtsassessorin, Geschäftsführerin Deutscher Juristinnenbund, Berlin.

\title{
Verkannte Vordenkerinnen
}

\section{Frauen als Objekte und als Gestalterinnen in der historischen Entwicklung des Strafrechts}

Ein Beitrag über die historische Entwicklung des Strafrechts ließe sich, je nachdem, wie „Strafrecht“ definiert wird, sehr ausufernd oder eher pointiert fassen. Denn wäre unter „Strafrecht“ jede soziale Reaktion auf begangenes Unrecht nach bestimmten Regeln zu verstehen, müsste eine Darstellung selbst für den deutschen Bereich streng genommen mit dem römischen Recht, spätestens aber mit dem Sanktionssystem der germanischen Stämme beginnen. Schon bevor es ein staatliches Strafmonopol gab, ahndeten die germanischen Sippen (ähnlich wie die altrömischen Familienverbände) begangenes Unrecht durch Fehde oder Buße. ${ }^{1}$ Bereits hier ließen sich Aspekte der Genderforschung fokussieren: Wurde eine Person getötet, entging der Schädiger der Blutfehde, wenn er als Buße ein Wergeld entrichtete. Nun waren die Wergeldkataloge in den verschiedenen Stammesrechten (sog. leges barbaorum) durchaus unterschiedlich gestaltet. Bisweilen war - ähnlich wie später im Sachsenspiegel, dem bekanntesten Rechtsbuch des Hochmittelalters das Wergeld für Frauen knapper bemessen als für Männer. Diese vermeintliche Geringschätzung der Frau ließe sich jedoch kulturhistorisch leicht erklären, wenn angesichts der kriegerischen Völkerwanderungszeit auf die Waffenfähigkeit abgestellt würde. Denn bei einem näheren Blick zeigt sich, dass ein ähnlich niedriges Wergeld auch bei dem nicht waffenfähigen Mann anzusetzen war. ${ }^{2}$ Darüber hinaus gibt es verschiedene Stammesrechte, in denen das Wergeld für die Tötung einer schwangeren Frau mit 700 Schillingen beinahe dem eines Bischofs entsprach. ${ }^{3}$ Bei anderen Völkerschaften wird für Frauen sogar ein höheres Wergeld als für den Mann festgesetzt, um den fehlenden Selbstschutz durch die erhöhte Abschreckung in Form der Buße zu ersetzen. ${ }^{4}$

Schon dieses kurze Beispiel zeigt, wie differenziert sich über die Stellung der Frau im historischen Strafrecht diskutieren ließe, wie viele Vor- oder Nachteile aus der Analyse alter Rechte oder Rechtsbräuche herzuleiten wären. Der Blick auf die schriftlich überlieferten Rechtsquellen wie etwa die vorgenannten Leges oder die später aufgezeichneten Rechtsspiegel lässt jedoch eine grundsätzliche Schlechterstellung der Frau in strafrechtlichen Belangen nicht immer erkennen. Die strafrechtlichen Bestimmungen sind schon damals oft geschlechtsneutral formuliert: „Wer des Nachts Korn stiehlt, ist des Galgens schuldig“. 5 Ob Mann oder Frau spielte keine Rolle. Wenn überhaupt „frauenpolitische“ Fragen an diese frühe Zeit herangetragen werden wollten, dann

1 Fehde und Buße sind typische Begriffe der germanistischen Rechtsgeschichte, hierzu Hinrich Rüping/Günther Jerouschek, Grundriss der Strafrechtsgeschichte, 2007, S. 4 f. Für die Römer wäre nach dem Zwölftafelgesetz wohl die Bezeichnung „Vergeltung“ (durch die Agnaten, also die Verwandten) oder „Geldbuße“ (sowohl bei geringeren Vergehen als auch im Fall der gütlichen Einigung, Tafel VIII, 2) angemessen, Stephan Meder, Rechtsgeschichte, 2008, S. 29-33.

2 Davon berichtet etwa Felix Dahn 1881 in seinem Aufsatz „Das Weib im altgermanischen Recht und Leben“, S. 3; das Dokument ist insgesamt ein interessant zu lesender Nachweis dafür, dass nicht alle Rechtslehrer dieser Zeit (der Verfasser war Ordinarius in Königsberg) erklärte Frauenfeinde gewesen waren.

3 So etwa in der Lex ribvaria (vor 625, nach Kroeschell, 1, $47 \mathrm{f}$.$) .$

4 So etwa in der Lex Baiuwariorum.

5 Sachsenspiegel, Landrecht II, 39, 1.

\section{Dr. Andrea J. Czelk}

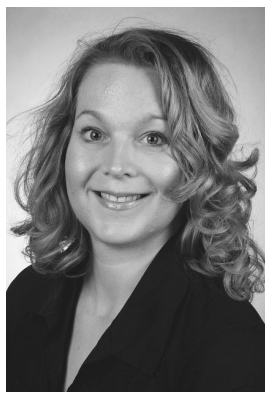

Akademische Rätin an der Juristischen Fakultät der Universität Hannover 
müssten sie an andere Gesichtspunkte anknüpfen, etwa an ein zum Nachteil der Frauen verlagertes Unrechtsbewusstsein. Dies könnte sich beispielsweise dann ergeben, wenn Delikte, deren Opfer regelmäBig Frauen sind - zu denken ist hier an die Vergewaltigung - zu mild oder gar nicht bestraft worden wären. Maßstab wiederum könnte jedoch nur unser heutiges Verständnis von Recht und Unrecht sein. $\mathrm{Ob}$ es der damaligen Zeit gerecht würde oder ob es sich um eine unangemessene Rückinterpretation handelte, verdiente eine längere Würdigung als in einem kurzen Artikel möglich ist.

Indiz für die Stellung der Frau im Strafrecht ist natürlich auch die Ahndung von Straftaten, bei denen nur weibliche Täter in Frage kamen oder Frauen verhältnismäßig häufig verurteilt wurden. Dies rückt die Hexerei, die Kindstötung und die Abtreibung in den Blickpunkt. Während Hexerei als „Delikt“ mit der Aufklärung abgeschafft wurde, haben die beiden zuletzt genannten Tatbestände Politik und Frauenbewegung noch weit in das 20.

\section{Die Hexerei als klassisches Beispiel frauenfeindlicher Ver- folgung war weniger ein Problem der Strafgesetze als viel- mehr der örtlichen Strafpraxis, des Denunziationsverhal- tens und des Irr- und Aberglaubens der Bevölkerung, aus deren Mitte die meisten Beschuldigungen hervorgingen.}

Jahrhundert beschäftigt. Die Diskussion - das zeigen auch immer aktuelle Debatten zu den neu auftretenden, atypischen Fällen der Kindstötung - ist zudem nicht abgeschlossen.

Selbst eine strafrechtshistorische Analyse lediglich derjenigen Deliktsgruppen, in denen Frauen typischerweise Täter oder Opfer sind, wäre so „ausufernd“ wie im ersten Satz des Beitrages angekündigt, wenn sie tatsächlich bis zum geltenden Recht verfolgt werden sollte. Eine Ausnahme lässt sich hier für die Hexerei machen, um neue Differenzierungsansätze aufzuzeigen:

Die Hexerei als klassisches Beispiels frauenfeindlicher Verfolgung war weniger ein Problem der Strafgesetze als vielmehr der örtlichen Strafpraxis, des Denunziationsverhaltens und - in regional variierenden Erscheinungsformen - des Irr- und Aberglaubens der Bevölkerung ${ }^{6}$, aus deren Mitte die meisten Beschuldigungen hervorgingen. Denn dass ausschließlich oder hauptsächlich Frauen Hexerei oder Schadenszauber begehen konnten, ist den wenigsten schriftlichen Quellen zu entnehmen. Vielmehr waren die meisten relevanten rechtlichen Bestimmungen $^{7}$ geschlechtsneutral und hätten ebenso gut auf Männer angewandt werden können.
Erst der Dominikanermönch Heinrich Kramer hat im berüchtigten Malleus Maleficarum ${ }^{8}$ die Hexerei eindeutig den Frauen zugewiesen; seinem Werk blieb indes die offizielle Anerkennung durch die Kirche versagt. Warum im deutschsprachigen Raum eine Vielzahl der Verurteilten tatsächlich Frauen waren, ist damit nicht abschließend geklärt. Denn allein die psychische Struktur verschiedener Kleriker (Schlagwort: „Angst vor der Frau“") wird als Begründung nicht genügen, solange in anderen europäischen Gebieten - z.B. in Island - vorrangig Männer als „Hexer“ verurteilt wurden. ${ }^{9}$ Dieser Umstand lenkt die Aufmerksamkeit auf das Denunziationsverhalten der Bevölkerung. Warum es aber überwiegend Frauen waren, die - oft genug von anderen Frauen - denunziert wurden, wird die Forschung noch lange beschäftigen. Möglicherweise liegen hier die brauchbarsten Erklärungsansätze in der Ethnologie, die Ursachen im alten Volksglauben findet, der je nach Region eher Frauen als Männer mit Zauberei in Verbindung brachte. ${ }^{10}$ Auch mag die tatsächliche Verbreitung des „Hexenhammers" und darauf basierende Predigten die Fokussierung auf die Frau als Täterin im Volk verstärkt haben, obschon, wie oben erwähnt, nicht nur die Kirche, sondern auch die Obrigkeit vielerorts Skepsis gegenüber dessen Thesen walten ließ. Vereinzelt wurden Kramers Vorstellungen, insbesondere das Verständnis der „Häckse“ als ausschließlich weibliche Person, dann nämlich doch übernommen, so z.B. vom Landvogt Tengler im „Augsburger Layenspiegel“ von $1511 .{ }^{11}$ Allerdings war in der Constitutio Criminalis Carolina (CCC), der Peinlichen Gerichtsordnung Kaiser Karls des V. von 1532, die erstmals Strafprozess und Strafmaß für das gesamte Reich regelte, die Formulierung des Tatbestandes in Artikel 109 wiederum eindeutig geschlechtsneutral: „item so jemandt den leuten durch zauberey schaden oder nachtheyl zufügt, soll mann straffen vom leben zum todt (...)“.

Diese Fragen müssen in einem kursorischen Abriss offen bleiben; im Detail bietet die Forschung eine

6 Ina Ahrendt-Schulte, Hexenprozesse, in: Ute Gerhard (Hrsg.), Frauen in der Geschichte des Rechts, S. 199-220. Sie nennt auch anschauliche Beispiele für den oben erwähnten Fall (S. 205).

7 So etwa in der Carolina, vgl. Art. 109.

8 Dt. „Hexenhammer“. Malleus Maleficarum 1487 von Heinrich Kramer (Institoris), Nachdruck der Erstausgabe von 1487 mit Bulle und Approbatio, 1992.

9 Rita Voltmer, Witch Hunts, in: Richard M. Golden (Hrsg.), Encyclopedia of Witchcraft, Bd. 4, 2006, S. 12091215 (S. 211); dies., Vom getrübten Blick auf die neuzeitliche Hexenverfolgung - Versuch einer Klärung, in: Forschungsgruppe Weltanschauungen Deutschland, Textarchiv TA-2006-12.

10 So etwa für Island, s.o. Fn. 9.

11 Ullrich Tengler, Der neu Layenspiegel, Augsburg 1511, S. 140-145. 
Vielzahl interessanter Ideen und Denkanstöße, ohne dass auch nur ein vorläufiger Abschluss der Diskurse abzusehen wäre. Beendet wurden die Verfolgungen schließlich im Zuge der strafrechtlichen Aufklärung im 18. Jahrhundert, als sich die Lehre von der Straflosigkeit der Hexerei allgemein durchsetzte. Schon 1631 hatte Friedrich von Spee die Hexerei als prozessuale Schimäre bezeichnet, deren Existenz sich ausschließlich auf den Einsatz von Folter und den Hexenwahn der Verfolger begründe. ${ }^{12}$ Thomasius entlarvte sie schließlich als Erfindung der „Päbstischen Pfaffen“13. Mit der Aufklärung wurde zudem die Folter hinterfragt und schließlich im 19. Jahrhundert vom Beweisprozess abgelöst. Zudem verschwanden die teilweise drakonisch anmutenden Strafmaßnahmen der CCC wie das Begraben bei lebendigem Leib und das Pfählen aus der Strafpraxis.

Bisher sind Frauen als Objekte, wenn nicht gar als Opfer der jeweiligen Strafrechtspflege beschrieben worden. Tatsächlich aber beginnt mit der Epoche der Strafrechtskodifikationen eine Phase, in der Frauen immer stärker versuchen, die Gesetze und das Strafverfahren aktiv zu gestalten. Der Einsatz der älteren bürgerlichen Frauenbewegung für eine - aus frauenpolitischer Sicht - gerechtere Fassung der Strafgesetze vor allem bei den oben genannten wichtigen Delikten der Abtreibung, der Kindstötung und den Sittlichkeitsdelikten hat in der Wissenschaft lange nicht die Anerkennung erfahren, die er verdient. Die Forderungen der Frauenbewegung folgen allerdings den ersten bedeutenden Kodifikationen des Strafrechts zeitlich nach. Die Aufklärung hatte, wie oben erwähnt, den Boden für eine neue Struktur des Strafrechts bereitet. In der Folge bemühten sich die deutschen Territorialstaaten, Gesetze zu schaffen, die dem modernen Verständnis angemessen waren. Die Straftatbestände sollten klar gefasst und - verglichen mit den älteren Ordnungen - mit relativ „humanen“ Strafen geahndet werden. Sanktionen waren etwa die Geldstrafe, die Haft (Zuchthaus-, Festungs- und Gefängnishaft) sowie die Todesstrafe bei Mord und „Hochverrath“. Der Strafprozess wurde nun getrennt geregelt und basierte auf dem „Beweisrecht“, das im Wesentlichen unserem heutigen Verständnis entspricht. Von zentraler Bedeutung ist das Strafgesetzbuch für die preußischen Staaten von 1853, da das später kodifizierte Strafgesetzbuch für den Norddeutschen Bund in weiten Teilen darauf basiert. Dieses zuletzt genannte Gesetzgebungswerk war dann wiederum Grundlage für das Reichsstrafgesetzbuch von 1871 .

Dass sich zum Reichsstrafgesetzbuch von 1871 noch keine Forderungen der Frauenbewegung fin- den, folgt nicht aus mangelndem Engagement. Die Frauenbewegung in Deutschland steckte 1871 vielmehr in den sprichwörtlichen „Kinderschuhen“. Es existierten zwar erste Vereine, und Vordenkerinnen wie Hedwig Dohm und Louise Otto-Peters begannen, mit rhetorisch aufrüttelnden Schriften auf die Benachteiligung des sog. „schwachen Geschlechts“ aufmerksam zu machen. Doch fehlte diesen Aktivitäten ein geeigneter Ansatzpunkt, um die patriarchalischen Strukturen zu durchbrechen. Ein solcher fand sich dann schließlich in den 1890er Jahren in

\section{Der Einsatz der älteren bürgerlichen Frauenbewegung für eine gerechtere Fassung der Strafgesetze hat in der Wissenschaft lange nicht die Anerkennung erfahren, die er verdient.}

den ersten Entwürfen zum neuen Bürgerlichen Gesetzbuch. Das geplante Familienrecht benachteiligte die Frauen nach wie vor etwa bei der Frage nach der sog. „elterlichen“, in Wahrheit jedoch rein „väterlichen “ Gewalt, im Güterrecht und bei den allgemeinen Ehewirkungen. ${ }^{14}$

Zugleich erhielten die Frauenvereine mit fortschreitender Zeit mehr Zulauf. Sie hatten nun die Schlagkraft, um gezielt für eine bessere rechtliche Stellung der Frauen zu streiten und nahmen den öffentlichen Kampf gegen die „patriarchalischen Gesetzgeber“ auf, indem sie Petitionen an den Reichstag sandten und in Agitationen gegen die Entwürfe mobilmachten. Nachdem das BGB 1900 ohne Berücksichtigung der Frauenforderungen in Kraft getreten war, nutzten die nunmehr erstarkten und unter dem Bund Deutscher Frauenvereine (BDF) zusammengeschlossenen Verbände ihre Erfahrung und wandten ihr Interesse verstärkt strafrechtlichen Fragen zu. Diese Bemühungen trafen zeitlich mit Reformbestrebungen der Strafrechtswissenschaft und der Gesetzgebung zusammen. 1909 entstand ein erster offizieller Reformentwurf.

Zeitgleich legt der BDF die auf der Generalversammlung in Breslau beschlossene Petition zum Reichsstrafgesetzbuch vor. Federführend war Camilla Jellinek, die sowohl die Begründungen als auch die Paragrafenfassungen - mit Ausnahme des \ $218 \mathrm{RStGB}^{15}$ - bearbeitete. Dieser Entwurf, der in seiner Präzision weder von Julie Eichholz‘ Vorarbeiten noch von nachfolgenden Reformvorschlägen

\footnotetext{
12 Hinrich Rüping/Günther Jerouschek, Grundriss der Strafrechtsgeschichte, 2007, Rn. 149.

13 Ebd. Rn. $172 \mathrm{f}$.

14 Zusammenfassend: Czelk, Privilegierung und Vorurteil, 2005, S. 39-43 m.w.N.

15 Alle folgenden Paragrafen sind solche des Reichsstrafgesetzbuches.
} 
erreicht wird, stellte eine ähnliche Überlegung voran, wie sie auch diesem Artikel zugrunde liegt. ${ }^{16}$ Camilla Jellinek konstatierte ein grundsätzliches Reformbedürfnis bei den Delikten, bei denen allein oder überwiegend Frauen als Täter in Frage kamen (Abtreibung, Kindstötung), in denen sie regelmäßig Opfer waren (Sittlichkeitsdelikte) oder in denen die familienrechtliche Vorherrschaft des Mannes in der Ehe ihren strafrechtlichen Widerpart fand (Ehebruch, Duell als Verteidigung der männlichen Ehre). Zudem sollten die Frauen zunehmend als Juristinnen oder wenigstens als Schöffinnen am Strafverfahren mitwirken dürfen.

Im Detail folgten aus dem Entwurf viele, in den folgenden Jahren stets aktuelle Vorschläge: Die Abtreibung (\$218) sollte - aus Sicht Camilla Jellineks - gänzlich straffrei bleiben. Überzeugt hatte sie das Elend der unteren sozialen Schichten und nicht zuletzt die eheliche Pflicht, die angesichts der fehlenden Empfängnisverhütung Frauen zu mehr Schwangerschaften zwang, als sie gesundheitlich verkraften konnten. Diese Forderung war dem BDF in der Mehrheit zu radikal; in der Abstimmung auf der Breslauer Generalversammlung unterlagen die Befürworterinnen der Abtreibungsfreiheit knapp. ${ }^{17}$ Stattdessen wurde einer Indikationslösung der Vorzug gegeben, die für die Petition von Katharina Scheven ausformuliert wurde. ${ }^{18} \mathrm{Im}$ historischen Kontext ist auch diese Indikationslösung aus dem Jahr 1909 durchaus als fortschrittlich anzusehen, entsprach sie doch in allen wesentlichen Punkten der Fassung, die schließlich von 1976 bis 1990 geltendes Recht war. Die Frauenbewegung war insofern selbst in einem gemäßigten Reformvorschlag den Vorstellungen des historischen Gesetzgebers weit voraus.

$\mathrm{Zu} \ 217$ forderte der BDF, dass die uneheliche Mutter, die ihr Kind unmittelbar nach der Geburt tötete, noch milder bestraft wurde, als es der Privilegierungstatbestand ohnehin vorsah. Jellinek begründete dies mit der unzureichenden Absicherung von Mutter und Kind durch das Unehelichenrecht des BGB und eine fehlende öffentliche Versorgung. Außerdem sollte der Vater als „mittelbarer Täter“ gleichfalls strafbar sein, wenn er die Frau während der Schwangerschaft weder menschlich noch finanziell unterstützte. ${ }^{19}$

Obschon sich die Forderung nach der Strafbarkeit des Vaters in $\$ 217$ nicht durchsetzen konnte, fand der Gedanke, der Mann müsse sich seiner Verantwortung gemäß verhalten, doch Eingang in das Strafgesetzbuch. Auch heute macht sich zumindest derjenige nach $\mathbb{} 170$ strafbar, der Unterhaltszahlungen verweigert, obwohl er zu deren Leistung in der Lage wäre.

Im Sexualstrafrecht plädierte der BDF für eine Ausweitung der Missbrauchstatbestände auf Ausbilder und Arbeitgeber. ${ }^{20}$ $\mathrm{Zu}$ Recht findet sich dieser Gedanke auch im heutigen Strafgesetzbuch. Außerdem hatte Carl Bulling, ein der Frauenbewegung nahestehender Jurist, schon 1895 die Strafbarkeit der Vergewaltigung in der Ehe gefordert. ${ }^{21}$ Diese Ansicht war dem Gesetzgeber bis 1998 und so manchem Vertreter der Rechtswissenschaft auch darüber hinaus zu fortschrittlich. Noch 1999 formulierte etwa Schroeder, es handele sich bei der Ver- gewaltigung in der Ehe doch nicht um eigentliches Vergewaltigungsunrecht, sondern um ein „Selbsthilferecht des Mannes“.22

Am Ende der Breslauer Generalversammlung stand ein Reformentwurf des BDF, der deutlich mehr mit dem modernen Strafgesetzbuch gemein hatte als mit dem damals geltenden Recht, an dem er sich orientierte. Die Reaktionen der Gesetzgeber und teilweise auch der Rechtsgelehrten waren zunächst negativ. Dies galt insbesondere auch für die Zulassung von Frauen zum Richteramt. ${ }^{23}$ Allerdings gelang es im Kaiserreich nicht, die Strafrechtsreform von amtlicher Seite aus abzuschließen.

Umso bedeutender ist die Anerkennung, die Protagonistinnen wie Camilla Jellinek in der Weimarer Zeit genossen haben. Gustav Radbruch hat nicht nur den ideellen Einsatz dieser Frauen honoriert, sondern auch inhaltlich bei vielen Fragen Zustimmung signalisiert. ${ }^{24}$ Zwar fand die Reform auch in der Weimarer Republik keinen Abschluss. Aber die Kommission, die schließlich in den 1950er Jahren über die große Strafrechtsreform beriet, war überwiegend mit ehemaligen Mitarbeitern des Weimarer Justizministeriums besetzt. ${ }^{25}$ Daher ist nicht auszuschließen, dass letztlich neuere Bestimmungen unmittelbar auf die Denkanstöße zurückgehen, die der BDF 1909 das erste Mal formuliert hat.

Wissenschaftlich wird sich ein solcher Zusammenhang kaum beweisen lassen. Zu Vieles ist auch auf andere Einflüsse zurückführen. Festzuhalten bleibt aber, dass die Frauenbewegung, als sie einmal ihre Kraft als Reformbewegung erkannt hatte, mit ihren Forderungen dem heute geltenden Recht deutlich näher und ihrer Zeit damit weiter voraus war, als es die (männlichen) Gesetzgeber damals für möglich hielten.

16 Camilla Jellinek, Petition des BDF zum Strafgesetzbuch, 1909, S. 3 f.

17 Czelk, Frauenrecht und Mutterschutz, in: Meder/Duncker/Czelk, Frauenrecht und Rechtsgeschichte, S. 351-366, 361 m.w.N.

18 Katharina Scheven, in: Petition des BDF zum Strafgesetzbuch, 1909, S. $45-52$.

19 Camilla Jellinek, Petition des BDF zum Strafgesetzbuch, 1909, S. 43-45.

20 Ebd. S. 8-10.

21 Carl Bulling, Die deutsche Frau und das bürgerliche Gesetzbuch, S. 146.

22 Friedrich-Christian Schroeder, Die Revolution des Sexualstrafrechts, in: JZ 1999, S. 827-833, (S. 828).

23 Hinweise und eine Reaktion der Frauenbewegung u.a. bei Helene Lange, Die ungeeignete Schöffin, in: Die Frau 1924/25, S. 268-270.

24 Das zeigt sich nicht zuletzt im Entwurf von 1922 (auch im E 25), in dem Radbruch ähnlich wie Jellinek in der Petition des BDF eine Strafbarkeit des Mannes wegen Pflichtversäumnisses der Frau gegenüber normiert (Stichwort: Unterhalt) und die Kindstötung der verlassenen Frau als schweren Erfolg einarbeitet.

25 Hubert Treiber, Die „rückwärtsgewandte“ Expertenreform - Innenansichten zur Großen Strafrechtsreform der 5oer Jahre, in: Festschrift für Gagnér, 1996, S. 229-273, zit. 260-265. 